\title{
Double charmonium production at B factories within light cone formalism
}

\author{
V.V. Braguta* \\ Institute for High Energy Physics, Protvino, Russia \\ E-mail: braguta@mail.ru
}

\begin{abstract}
This report is devoted to the study of the processes $e^{+} e^{-} \rightarrow J / \Psi \eta_{c}, J / \Psi \eta_{c}^{\prime}, \psi^{\prime} \eta_{c}, \psi^{\prime} \eta_{c}^{\prime}$ within light cone formalism. It is shown that the results of the calculation of the cross sections of these processes are in agreement with Belle and BaBar experiments. It is also shown that relativistic and radiative corrections to the cross sections play crucial role in the achievement of the agreement between the theory and experiments.
\end{abstract}

8th Conference Quark Confinement and the Hadron Spectrum September 1-6 2008

Mainz, Germany

* Speaker. 
Double charmonium production at B-factories has been one of the most challenging problem in quarkonium physics for many years. This problem appeared after the measurements of the cross sections of the processes $e^{+} e^{-} \rightarrow J / \Psi \eta_{c}, J / \Psi \eta_{c}^{\prime}, \psi^{\prime} \eta_{c}, \psi^{\prime} \eta_{c}^{\prime}, J / \Psi \chi_{c 0}, \psi^{\prime} \chi_{c 0}$ at Belle [1, 2] and BaBar [3] collaborations, which were approximately by an order of magnitude larger than the leading order nonrelativistic QCD (NRQCD) predictions $[4,5,6]$. There were many attempts to resolve this discrepancy. Lately, it was shown that the agreement between theory and experiment can be achieved within NRQCD if one takes into account radiative and relativistic corrections to the cross sections $[7,8]$.

Another systematic approach to the study of hard exclusive processes is light cone formalism (LC) $[9,10]$. Within this approach the amplitude of hard exclusive process can be separated into two parts. The first part is partons production at very small distances, which can be treated within perturbative QCD. The second part is the hadronization of the partons at larger distances. This part contains information about nonperturbative dynamic of the strong interactions. For hard exclusive processes it can be parameterized by process independent distribution amplitudes (DA), which can be considered as hadrons' wave functions at light like separation between the partons in the hadron. It should be noted that within LC one does not assume that the mesons are nonrelativistic. This approach can equally well be applied to the production of light and heavy mesons, if the DAs of the produced meson are known. For this reason, one can hope that within this approach one can study the production of excited charmonia states.

In this report the results of the calculation of the processes $e^{+} e^{-} \rightarrow J / \Psi \eta_{c}, J / \Psi \eta_{c}^{\prime}, \psi^{\prime} \eta_{c}, \psi^{\prime} \eta_{c}^{\prime}$ within LC will be briefly described. The details of this calculation can be found in paper [14]. It will be shown that with the models of DAs proposed in papers $[11,12,13]$ LC predictions are in agreement with the results obtained at Belle and BaBar experiments.

Now let us consider the amplitude of the process $e^{+} e^{-} \rightarrow V P$, where $V=J / \Psi, \psi^{\prime}$ and $P=$ $\eta_{c}, \eta_{c}^{\prime}$. Two diagrams that give contribution to the amplitude of this process are shown in Fig. 1. The other two can be obtained from the depicted ones by the charge conjugation. The amplitude of
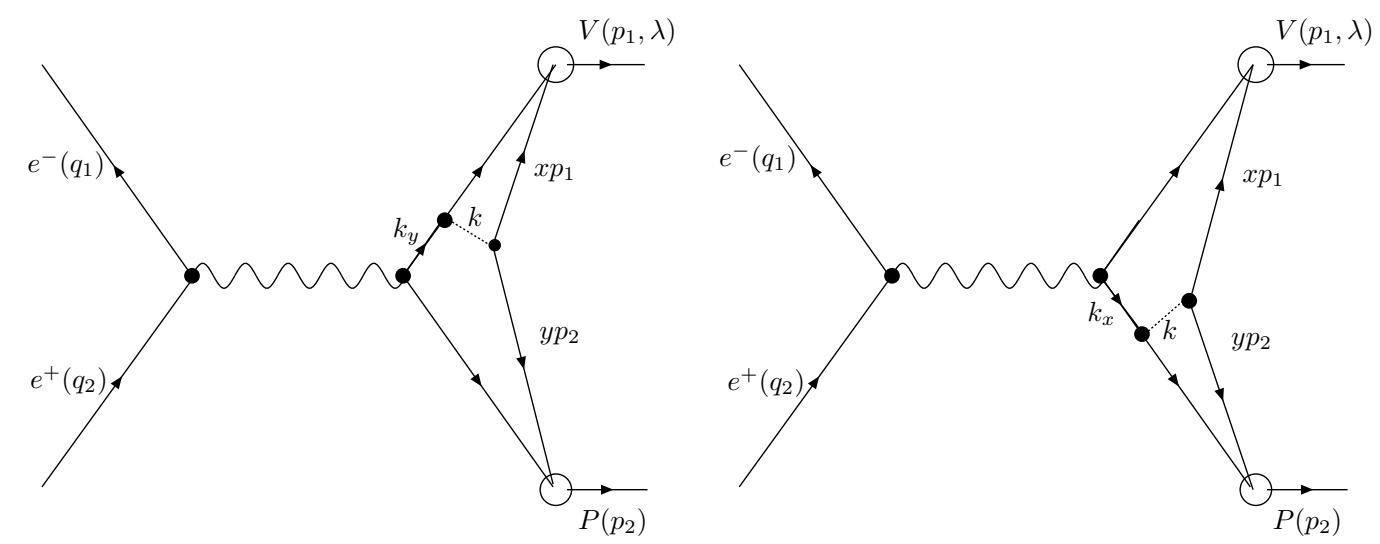

Figure 1: The diagrams that contribute to the process $e^{+} e^{-} \rightarrow V\left(p_{1}, \lambda\right) P\left(p_{2}\right)$ at the leading order approximation in the strong coupling constant. 


\begin{tabular}{|c|c|c||c|c||c|c||c|}
\hline$H_{1} H_{2}$ & \multicolumn{2}{|c||}{$\sigma_{E x p} \times B_{H_{2} \rightarrow \text { charged }>2}(\mathrm{fb})$} & \multicolumn{2}{c||}{$\sigma_{L O ~ N R Q C D}(\mathrm{fb})$} & \multicolumn{2}{c|}{$\sigma_{N R Q C D}(\mathrm{fb})$} & $\sigma_{L C}(\mathrm{fb})$ \\
\hline & Belle[2] & BaBar[3] & {$[4]$} & {$[5]$} & {$[7]$} & {$[8]$} & \\
\hline$\psi(1 S) \eta_{c}(1 S)$ & $25.6 \pm 2.8 \pm 3.4$ & $17.6 \pm 2.8_{-2.1}^{+1.5}$ & $3.78 \pm 1.26$ & 5.5 & 20.4 & $17.6_{-8.3}^{+10.7}$ & $14.4_{-9.8}^{+11.2}$ \\
\hline$\psi(2 S) \eta_{c}(1 S)$ & $16.3 \pm 4.6 \pm 3.9$ & - & $1.57 \pm 0.52$ & 3.7 & - & - & $10.4_{-7.8}^{+9.2}$ \\
\hline$\psi(1 S) \eta_{c}(2 S)$ & $16.5 \pm 3.0 \pm 2.4$ & $16.4 \pm 3.7_{-3.0}^{+2.4}$ & $1.57 \pm 0.52$ & 3.7 & - & - & $13.0_{-11.0}^{+12.2}$ \\
\hline$\psi(2 S) \eta_{c}(2 S)$ & $16.0 \pm 5.1 \pm 3.8$ & - & $0.65 \pm 0.22$ & 2.5 & - & - & $9.0_{-8.5}^{+9.7}$ \\
\hline
\end{tabular}

Table 1: The second and third columns contain experimental results measured at Belle and Babar experiments. The $\mathrm{Br}_{\mathrm{H}_{2} \rightarrow \text { charged }>2}$ means the branching ratio of the decay of the hadron $\mathrm{H}_{2}$ into two charged particles. In the fourth and fifth columns the results of the leading order NRQCD obtained in papers [5, 4] are shown. The NRQCD results obtained with inclusion of radiative and relativistic corrections $[7,8]$ are shown in columns six and seven. Last column contains the values of the cross sections obtained in this paper.

the process involved can be written in the following form:

$$
M=-4 \pi \alpha \frac{{ }^{-} u(k) \gamma_{\mu} u\left(k_{2}\right)}{s}\left\langle V\left(p_{1}, \lambda\right) P\left(p_{2}\right)\left|J_{\mu}^{e m}\right| 0\right\rangle,
$$

where $\alpha$ is the electromagnetic coupling constant, ${ }^{-} u(k), u\left(k_{2}\right)$ are the electron and positron bispinors, $\sqrt{s}$ is the invariant mass of $e^{+} e^{-}$system, $J_{\mu}^{e m}$ is the electromagnetic current. The matrix element $\left\langle V\left(p_{1}, \lambda\right) P\left(p_{2}\right)\left|J_{\mu}^{e m}\right| 0\right\rangle$ can be parameterized by the only formfactor $F(s)$ :

$$
\left\langle V\left(p_{1}, \lambda\right) P\left(p_{2}\right)\left|J_{\mu}^{e m}\right| 0\right\rangle=i q_{c} F(s) e_{\mu v \rho \sigma} \varepsilon_{\lambda}^{v} p_{1}^{\rho} p_{2}^{\sigma},
$$

where $q_{c}$ is the charge of $c$ quark, $\varepsilon_{\lambda}^{v}$ is the polarization vector of the meson $V\left(p_{1}, \lambda\right)$. The cross section of the process under consideration can be written as follows

$$
\sigma\left(e^{+} e^{-} \rightarrow V P\right)=\frac{\pi \alpha^{2} q_{c}^{2}}{6}\left(\frac{2|\mathbf{p}|}{\sqrt{s}}\right)^{3}|F(s)|^{2} .
$$

In the last formula $\mathbf{p}$ is the momentum of the meson $V$ in the center mass frame of the final mesons.

The analytical expression for the formafactor $F(s)$ obtained within LC and the detail of the calculation of the cross sections of the processes under study can be found in paper [14]. In this report only the results of this calculation are given. These results are presented in Table I. The second and third columns contain experimental results measured at Belle and Babar experiments. In the fourth and fifth columns the results of the leading order of NRQCD approach obtained in papers [5, 4] are shown. The NRQCD results obtained with inclusion of radiative and relativistic corrections $[7,8]$ are shown in columns six and seven. Last column contains the values of the cross sections obtained in this paper.

In addition to the calculation of the cross sections of the processes under study it is interesting to answer the question: why LC predictions are much greater than the leading order NRQCD predictions. Numerical results of the calculation shows that large disagreement between LC and the leading order NRQCD predictions can be attributed to large contribution of relativistic and radiative corrections. From this results one can draw a conclusion that in hard exclusive processes relativistic 
and radiative corrections play very important role and the consideration of such processes at the leading NRQCD approximation is unreliable. The results of this paper are in agreement with recent NRQCD study of the process $e^{+} e^{-} \rightarrow J / \Psi \eta_{c}[7,8]$ where the authors took into account relativistic and one loop radiative corrections.

\section{References}

[1] K. Abe et al. [Belle Collaboration], Phys. Rev. Lett. 89, 142001 (2002), [arXiv:hep-ex/0205104].

[2] K. Abe et al. [Belle Collaboration], Phys. Rev. D 70, 071102 (2004), hep-ex/0407009.

[3] B. Aubert [BABAR Collaboration], hep-ex/0506062.

[4] E. Braaten and J. Lee, Phys. Rev. D 67, 054007 (2003) [arXiv:hep-ph/0211085];

[5] K. Y. Liu, Z. G. He and K. T. Chao, Phys. Lett. B 557, 45 (2003) [arXiv:hep-ph/0211181];

[6] K. Y. Liu, Z. G. He and K. T. Chao, arXiv:hep-ph/0408141.

[7] Z. G. He, Y. Fan and K. T. Chao, Phys. Rev. D 75, 074011 (2007) [arXiv:hep-ph/0702239].

[8] G. T. Bodwin, J. Lee and C. Yu, Phys. Rev. D 77, 094018 (2008) [arXiv:0710.0995 [hep-ph]].

[9] G. P. Lepage and S. J. Brodsky, Phys. Rev. D 22, 2157 (1980).

[10] V. L. Chernyak and A. R. Zhitnitsky, Phys. Rept. 112, 173 (1984).

[11] V. V. Braguta, A. K. Likhoded and A. V. Luchinsky, Phys. Lett. B 646, 80 (2007) [arXiv:hep-ph/0611021].

[12] V. V. Braguta, Phys. Rev. D 75, 094016 (2007) [arXiv:hep-ph/0701234].

[13] V. V. Braguta, arXiv:0709.3885 [hep-ph].

[14] V. V. Braguta, arXiv:0811.2640 [hep-ph]. 